\title{
Bacterial community analysis for investigating bacterial transfer from tonsils to the pig carcass
}

Jakobsen, Anne Mette; Bahl, Martin lain; Buschhardt, Tasja; Hansen, Tina Beck; Al-Soud, Waleed Abu; Brejnrod, Asker D.; Sørensen, Søren J.; Nesbakken, Truls; Aabo, Søren

Published in:

International Journal of Food Microbiology

Link to article, DOI:

10.1016/j.ijfoodmicro.2019.02.003

Publication date:

2019

Document Version

Peer reviewed version

Link back to DTU Orbit

Citation (APA):

Jakobsen, A. M., Bahl, M. I., Buschhardt, T., Hansen, T. B., Al-Soud, W. A., Brejnrod, A. D., Sørensen, S. J., Nesbakken, T., \& Aabo, S. (2019). Bacterial community analysis for investigating bacterial transfer from tonsils to the pig carcass. International Journal of Food Microbiology, 295, 8-18.

https://doi.org/10.1016/j.ijfoodmicro.2019.02.003

\section{General rights}

Copyright and moral rights for the publications made accessible in the public portal are retained by the authors and/or other copyright owners and it is a condition of accessing publications that users recognise and abide by the legal requirements associated with these rights.

- Users may download and print one copy of any publication from the public portal for the purpose of private study or research.

- You may not further distribute the material or use it for any profit-making activity or commercial gain

- You may freely distribute the URL identifying the publication in the public portal 
1 Bacterial community analysis for investigating bacterial transfer from tonsils to the pig carcass

2

3 Anne Mette Jakobsen ${ }^{a}$, Martin Iain Bahla ${ }^{\mathrm{a}}$, Tasja Buschhardta ${ }^{\mathrm{a}}$, Tina Beck Hansen ${ }^{\mathrm{a}}$, Waleed Abu Al-Soud ${ }^{\mathrm{c}}$,

4 Asker D. Brejnrodc, Søren J. Sørensenc ${ }^{c}$, Truls Nesbakken ${ }^{\mathrm{b}}$ and Søren Aabo ${ }^{\text {a* }}$

5 a Technical University of Denmark - National Food Institute, Kemitorvet, Building 202, DK-2800 Kgs. Lyngby,

6 Denmark

7 b Norwegian University of Life Sciences, Faculty of Veterinary Medicine, Campus Oslo, Dept. of Food Safety

8 and Infection Biology, P. O. Box 8146 Dep., 0033 Oslo, Norway

9 c University of Copenhagen - Department of Biology, Section of Microbiology, Universitetsparken 15, DK-

102100 København $\varnothing$

$11{ }^{*}$ Corresponding author

12

Keywords: Pig slaughter, indicator, microbiota, 16S rRNA gene sequencing, cross-contamination 


\section{Abstract}

Tonsils in the oral cavity are an important source of contamination during pig slaughter, but have not received as much attention as faecal contamination. In the present study, ten pigs were sampled from tonsils, faeces and three different areas on each carcass. The samples were analysed by both culturing of Escherichia coli and Yersinia enterocolitica and by 16S rRNA gene sequencing to characterize the bacterial communities. Comparing culture data from deep tonsil tissue and tonsil surface showed similar numbers of E. coli but significantly higher numbers of $Y$. enterocolitica in the deep tissue samples. Microbiota analysis showed similar bacterial communities in the two sample types at phylum level, while comparison at genus level showed significant differences between the relative abundance of several genera in the two sample types. The finding of a significantly higher relative abundance of Yersinia in tonsil tissue compared to tonsil surface supported the culture analysis. The microbiota analysis also investigated characteristics of the bacterial community that could discriminate bacterial transfer from tonsils and faeces to the carcass during slaughter. The microbiota analyses demonstrated that Fusobacteria and Proteobacteria are the most abundant phyla in tonsils, while Firmicutes showed the highest relative abundance in faeces. The dominating phylum on carcasses was Proteobacteria. Besides Proteobacteria, the swabbing area on the forepart of the carcass, showed a higher relative abundance of Firmicutes and Fusobacteria compared to swabbing areas on the rear part and mid-section of the carcass. Principal coordinate analysis showed clear clustering of samples based on sample source (tonsils, faeces and carcass). Carcass swab samples from the forepart tended to cluster closer to the tonsil samples compared to carcass swab samples from the rear part and midsection. Identification of the genera Fusobacterium, Moraxella, Actinobacillus and non-E. coli genera of the family Enterobacteriaceae in carcass swabs could indicate tonsil contamination, while faecal contamination would more likely include higher prevalence of bacteria belonging to the class of Clostridia. The present study supports that it is possible to identify bacterial groups that are indicative for either tonsil or faecal carcass contamination. The level and composition of Enterobacteriaceae on the carcasses did, however, indicate that other sources of meat contamination than tonsils and faeces may be important, such as the process environment. 


\section{Introduction}

During pig slaughter, cross-contamination and transfer of bacteria can easily occur from e.g. the gastrointestinal tract or the oral cavity to the rest of the carcass (Borch et al., 1996). The risk of contamination from the gastrointestinal tract is commonly considered important, due to the very high bacterial load in this compartment. However, it is also important to consider the oral cavity, containing the palatine tonsils, as a source of bacterial contamination of the carcass. Until now, methods to distinguish between the various sources of bacterial contamination of carcasses during slaughter have been restricted to culture-based techniques. These have inherent low resolution due to limitations in culturing and typing of many bacterial groups leading to a lack in information on the relative contribution of the different sources.

The tonsils are known to be colonized by a diverse bacterial community, which includes both pathogenic and non-pathogenic species and serve the role of presenting the immune system to foreign substances including bacteria (Fredriksson-Ahomaa et al., 2009; Horter et al., 2003; Lowe et al., 2011). Bacterial colonisation of the tonsils may further facilitate entry into the animal (Horter et al., 2003). During slaughter and dressing of carcasses, it is common practise to recommend that the tonsils are not cut through (Anonymous, 2014). Consequently, the tonsils should follow the plucks (the red offal) and not remain on the head/carcass after evisceration, since the splitting procedure might lead to contamination of the head and potentially transfer of bacteria to other areas of the carcass. Specific pathogenic bacteria such as Salmonella enterica, Listeria monocytogenes, Campylobacter spp. and Yersinia enterocolitica have all previously been isolated from pig tonsils by use of culture-dependent methods (Fredriksson-Ahomaa et al., 2009; Horter et al., 2003; Nesbakken et al., 2003). Recently, the use of culture-independent DNA sequencing methods has provided much deeper knowledge concerning the natural microbial diversity of tonsils. Accordingly, Lowe et al. (2012) defined the 'core microbiome' of the palatine tonsils in pigs to include the bacterial families Pasteurellaceae, Moraxellaceae, Fusobacteriaceae, Veillonellaceae, Peptostreptococcaceae and Streptococcaceae.

In addition to pathogens as Salmonella spp. and Y. enterocolitica, often described in relation to pig slaughter, the tonsils have also been shown to contain a significant proportion of bacteria belonging to the phylum Fusobacteria, which include members known to have the potential of causing illness in both humans and animals (Aliyu et al., 2004; Fredriksson-Ahomaa et al., 2009; Langworth, 1977; Lowe et al., 2012). 
The main objective of the present study was to investigate whether bacterial transfer from tonsils and faeces to predefined areas of the pig carcass during pig slaughter could be elucidated by comparing systematic differences between bacterial communities in faeces and tonsils to the composition of the microbiota on the carcass. We further explored the possibility to identify bacterial communities, which can indicate the origin of the contamination. To enable this, the bacterial communities in samples from tonsils, faeces and three different areas on the carcass from the same pig were analysed by 16S rRNA gene sequencing. In parallel, specific bacterial species, namely $Y$. enterocolitica and $E$. coli, were also enumerated by culturing. Carcass contamination from the tonsils could differ, depending on whether tonsils are damaged or not during slaughter. Therefore, differences between the bacterial community on the surface and in the deep tissue of the tonsils were also investigated.

\section{Material and methods}

\subsection{Collection of samples}

The investigation was carried out during October and November 2013 and consisted of two sampling rounds. First, for comparison of the microbiota within the tonsil tissue and on the tonsil surface, respectively, tonsils were collected from pigs in two Norwegian slaughterhouses with a slaughter capacity of approximately 120 150 pigs per hour. Each slaughterhouse was visited once. Between three and 26 pigs were sampled from each of five different herds. Tongue and tonsils were taken from the pluck using sterile equipment just after evisceration and placed into sterile plastic bags. All samples were kept under cold conditions (approximately $5{ }^{\circ} \mathrm{C}$ ) in the slaughterhouse and during transport to the laboratory. A total of 35 tonsils were included for analysis following visual inspection of integrity in the laboratory.

Second, for the comparison of the microbial community of faeces, tonsils and three different carcass areas, samples were collected from ten additional pigs, two pigs from each of five herds, in a single Norwegean slaughterhouse. Tonsils were retrieved as described above. Faecal samples were collected by cutting off a $10-15 \mathrm{~cm}$ section of the rectum and placing it in a sterile plastic bag. For carcass swabs, sterile gauze swabs (4 ply) were placed in sterile stomacher bags and pre-moistened with $7 \mathrm{ml}$ peptone water $(1 \mathrm{~g}$ Bacto Peptone (Difco, 0118-17, Fisher Scientific, Denmark) and $8.5 \mathrm{~g} \mathrm{NaCl}$ per litre). Carcass swabs were taken just before cooling from three areas on one half of each carcass (Figure 1). Area A: approx. $400 \mathrm{~cm}^{2}$ located 
on the inner side of the rear section of the carcass and around the rectum, Area B: approx. $1,000 \mathrm{~cm}^{2}$ stretching from the forepart to the rear section on the inner side of the carcass along the backbone on the cut surface and area C: approx. $400 \mathrm{~cm}^{2}$ on the inner side of the forepart around the jaw and throat. The areas were selected due to the putative higher possibility of contamination from the oral cavity on area C, contamination from the saw cutting through the head on area B and contamination from the rectum on area A. A single swab sample was additionally taken from the saw used to cut the carcass. All samples were placed in a cooling box with cooling elements and kept cold until further analyses in the laboratory.

\subsection{Bacteriological analysis}

\subsubsection{Sample preparation}

In the laboratory, both palatine tonsils were removed from the root of the tongue using sterile instruments and transferred to a stomacher bag with filter (BagFilter® No. 8, WWR, Denmark). Peptone water was added to the stomacher bag $(1: 1 \mathrm{w} / \mathrm{v})$ and following $20 \mathrm{~s}$ of stomaching, the tonsils were removed from the stomacher bag and the liquid collected for analysis. To expose bacteria located in the deep tonsil tissue, the same tonsils were subsequently sliced thoroughly with a sterile scalpel. The slices were transferred to a new stomacher bag with filter. Peptone water was added (1:1 w/v) and the samples stomached for $20 \mathrm{~s}$ and liquid collected for analysis. For the samples from the ten pigs, tonsil surface was prepared for analysis as described above. Faeces were collected from the rectum sections and diluted with peptone water (1:10 w/v) and transferred to stomacher bags with filter, and stomached for $20 \mathrm{~s}$. For carcass and saw swabs, a volume of $20 \mathrm{ml}$ peptone water was added to the stomacher bags and samples were homogenised for $20 \mathrm{~s}$.

\subsubsection{Culture dependent analysis for Yersinia enterocolitica}

All samples were analysed quantitatively for $Y$. enterocolitica by direct plating on cefsulodin-irgasannovobiocin (CIN) agar plates (CM653 + SR109 Oxoid, Fisher Scientific, Denmark) and Y. enterocolitica chromogenic medium (YeCM) (CHROMagar, SmithMed, Norway). In parallel, ten-fold dilutions from all samples were enriched in irgasan-ticarcillin-potassium chlorate (ITC) broth (Sigma-Aldrich, Denmark) and analysed semi-quantitatively by plating on $\mathrm{CIN}$ and $\mathrm{YeCM}$ agar. The qualitative and the quantitative analysis followed essentially the standard procedures of NMKL $1173^{\text {rd }}$ edition but were modified by using YeCM as an additional indicative medium. 


\subsubsection{Identification and characterisation of Yersinia enterocolitica}

Presumptive colonies were sub-cultured on bromthymol-blue sucrose agar on which $Y$. enterocolitica produce yellow colonies due to the fermentation of sucrose. Further biotyping was based on the revised scheme of Wauters et al. (1987) using tests for esculin hydrolysis and indole production from tryptophan together with a test for urea formation on urea agar (Difco; 0283-02-6 Bacto Urea agar Base, 0140-01 Bacto Agar, Fisher Scientific, Denmark). Finally, isolates of Biotype 4 were serotyped by use of slide agglutination with commercial O:3 antiserum (Bio-Rad; Statens Serum Institute, Denmark).

\subsubsection{Culture analysis for Escherichia coli}

Samples obtained from tonsils, faeces and carcass swabs were analysed quantitatively for $E$. coli by use of the SimPlate method (AOAC International, 2005). From appropriate 10-fold dilutions, $1 \mathrm{ml}$ was placed in the centre of the plating device (SimPlate Devices, Part No: 65009-20, Biocontrol, Germany) and $9 \mathrm{ml}$ of blue mixed nutrient agar (Multi Test, Part No: 66008-500, Biocontrol, Germany) was added in the same spot. The sample was mixed gently in the SimPlate device according to the instruction of the supplier and the plates were incubated at $37^{\circ} \mathrm{C}$ for $24-28 \mathrm{~h}$. Wells with a change in colour from blue to red and fluorescent when exposed to UV-light at $366 \mathrm{~nm}$, were counted as positives. Quantification of $E$. coli per $\mathrm{ml}$ was calculated based on the most probable number (MPN) principle.

\subsection{Bacterial community analysis by $16 \mathrm{~S}$ rRNA gene sequencing}

From each sample, $10 \mathrm{ml}$ homogenate was transferred to a $15 \mathrm{ml}$ centrifuge tube and centrifuged at $10{ }^{\circ} \mathrm{C}$ for 10 min at $4500 \mathrm{rpm}$ (Beckman Coulter, Allegra X-22R centrifuge, Minnesota, US). The supernatant was discarded and the pellet was carefully re-suspended in $1 \mathrm{ml} \mathrm{LB}$ medium containing $15 \%$ glycerol. One $\mathrm{ml}$ of the cell suspension was transferred to a $1.5 \mathrm{ml}$ Eppendorf tube and stored at $-80^{\circ} \mathrm{C}$ until DNA extraction. DNA was extracted from the thawed cell suspensions using the PowerLyzer® PowerSoil ${ }^{\circledR}$ DNA Isolation Kit (Mo Bio Laboratories, Carlsbad, CA, US) following the manufacturer instructions, modified by using a Mixer Mill MM 300 (Retsch GmbH, Haan, Germany) for 10 min at 30 cycles per second for cell lysis. DNA extracts were kept frozen at $-20^{\circ} \mathrm{C}$ until further analyses (approx. after two months).

The 16S rRNA gene amplification procedure followed a two-step strategy, in which the first amplified the V3V4 variable regions from the 16S rRNA gene and the second attached Illumina sequencing sample specific adaptors. In the first PCR reaction, the universal primers PBU (5'-CCT ACG GGA GGC AGC AG-3') 
(Tulstrup et al., 2015) and PBR-long (5'-GGA CTA CCA GGG TAT CTA ATC-3') (developed in this study) were used to amplify approximately 466 base pairs (V3-V4 region) of the 16S rRNA gene. The reaction mixture consisting of $4 \mu \mathrm{l}$ 10X AccuPrime PCR Buffer II, $0.8 \cup$ AccuPrime Taq DNA Polymerase (Invitrogen, Life technologies, CA, US), $0.4 \mu \mathrm{M}$ primer PBU, $0.4 \mu \mathrm{M}$ primer PBR-long, $0.8 \mu \mathrm{g}$ bovine serum albumin (BSA) and $10 \mu \mathrm{l}$ template DNA, in a total volume of $40 \mu \mathrm{l}$ per sample. The DNA extracts from the faecal samples were diluted ten-fold before use as templates in the PCR reaction. The PCR program consisted of an initial denaturation at $94^{\circ} \mathrm{C}$ for $2 \mathrm{~min}$, followed by 30 cycles amplification at $94{ }^{\circ} \mathrm{C}$ for 20 sec, annealing at $58{ }^{\circ} \mathrm{C}$ for $20 \mathrm{sec}$ and extension at $68{ }^{\circ} \mathrm{C}$ for $1 \mathrm{~min}$, followed by a final extension at $68{ }^{\circ} \mathrm{C}$ for 5 min and cooling to $4{ }^{\circ} \mathrm{C}$. The size and quality of the PCR products were assessed by gel electrophoreses, and purified with QIAquick ${ }^{\circledR}$ PCR Purification kit (Qiagen, Valencia, CA, US) according to the manufacturer instructions. The DNA concentration of the purified PCR products was measured with a Qubit ${ }^{\circledR}$ fluorometer (Thermo Fisher Scientific) and adjusted to $5 \mathrm{ng} / \mu \mathrm{l}$ DNA for use as template for the second PCR amplification.

The second PCR amplification was carried out in 96-well microtiter plates using sequencing primers originally published by Yu et al. (2005) and modified by Sundberg et al. (2013) as follows: 341F (5'-CCT AYG GGR BGC ASC AG-3') and 806R (5'-GGA CTA CNN GGG TAT CTA AT-3'), both of which with 5' Illumina sequencing adaptors and index-tags as previously described (Mortensen et al., 2016; Thorsen et al., 2016). The reaction mix contained $2.0 \mu \mathrm{l}$ of the diluted amplicons (5 ng/ $\mu \mathrm{l}) 2 \mu \mathrm{l} 10 \mathrm{x}$ AccuPrime PCR Buffer II, 0.6U AccuPrime Taq DNA Polymerase (Invitrogen, Life technologies, CA, US) and $0.5 \mu \mathrm{M}$ fusion $341 \mathrm{~F}$ and $0.5 \mu \mathrm{M}$ fusion $806 \mathrm{R}$ in a total volume of $20 \mu \mathrm{l}$. The PCR was run as above, with exception of a reduced annealing temperature of $56^{\circ} \mathrm{C}$ and 15 amplification cycles.

\subsubsection{DNA sequencing}

The amplification products of the second PCR were purified with Agencourt AMPure XP Beads (Beckman Coulter Genomics, MA, US) following the manufacturer instructions using $0.7 \times$ volume beads and quantified as described above. Equimolar amounts of all the amplification products were pooled together in a single tube and concentrated using the DNA Clean \& Concentrator TM-5 Kit (Zymo Research, Irvine, CA, US) following the manufacturer instructions. The concentration of the pooled library was determined using the Quant-iT TM High-Sensitivity DNA Assay Kit and Qubit® fluorometer (Life Technologies) following the manufacturer instructions. Amplicon sequencing was performed on the Illumina MiSeq Desktop Sequencer 
182 (Illumina Inc., CA, US). For each run, a 1 - $5 \%$ PhiX DNA was added. All reagents used were from the 183 MiSeq Reagent Kits v2 (Illumina Inc., CA, US). Automated cluster generation and 250 paired-end 184 sequencing with dual-index reads were performed. Up to 192 samples were sequenced per run. Technical replicates of the sequencing were not carried out in this study. In parallel studies, the variation between technical replicates has been addressed and found to be minimal (data not shown).

\subsubsection{Bioinformatics on bacterial community data}

Raw fastq-files demultiplexed using the MiSeq Controller Software (Illumina Inc., CA, US) were processed with BioDSL (https://github.com/maasha/BioDSL). Primers and diversity spacers were identified and truncated with the function trim_primer(). Paired ends were mated using the function assemble_pairs(). Any sequences shorter than $100 \mathrm{bp}$ were discarded and remaining sequence reads were dereplicated using dereplicate_seq. The sequences were clustered at $97 \%$ identity with cluster_otus(), a wrapper around USEARCH ver. 7.0.1090 (Edgar, 2010). Chimeras were checked and removed with uchime_ref() against the GOLD database (Haas et al., 2011). The resulting sequences were classified using classify_seq against the Ribosomal Database Project (RDP) trainset9 database (032012) available from the Mothur website. Align_seq_mothur (Schloss et al., 2009), a wrapper around Mothurs align.seq() function for aligning Operational Taxonomic Units (OTUs) against an RDP template. The Mothur version used was v.1.33.3. Downstream processing of sequence data in the generated OTU table was performed in QIIME (Caporaso et al., 2010) using the core_diversity_analysis.py script. For diversity analysis, a phylogenetic tree was generated in QIIME using the make_phylogeny.py script based on a PyNAST alignment of all identified OTUs including an archaea for rooting. The OTU-table was first filtered to include only those OTUs annotated to Bacteria and to exclude OTUs with a number of sequences below $0.005 \%$ of the total number of sequences (Bokulich et al., 2012). Samples were rarefied to 10,000 reads per sample for analysis and those samples with fewer reads were excluded. The relative abundance at phylum level was calculated using the collapse_samples.py function. Phyla present in less than $1 \%$ in any sample type was compiled in the phyla 'other'. Alpha diversity, describing the bacterial diversity within a sample, was calculated for each sample by the Shannon index and species diversity (based on OTU richness). Assessment of beta diversity, describing the overall diversity in the community composition between sample types, was performed by Principal Coordinate Analysis (PCoA) based on unweighted UniFrac distances. Variation within and between 
211 the different sample types were calculated based on the unweighted UniFrac distances by use of the 212 make_distance_boxplots.py function. Heatmaps were used to visualize relative bacterial abundances at the 213 genus level for individual samples across sample types. The heatmaps were based on the relative abundance of the genera present in at least $35 \%$ of all samples, with filtering performed with the 215 filter_otus_from_otu_table.py script. Additionally, bacteria which could not be classified at the genus level were excluded from the heatmaps. Similarity between community composition at the operational taxonomic unit (OTU) level in tonsils, faeces and the three areas on the carcass was assessed using the shared_phylotypes.py script.

\subsection{Statistics}

For the culture data, statistics were conducted on $\log _{10}$ transformed data in the Microsoft Excel (2010) Data analysis add-in. A paired t-test was used for the comparison of the number of $E$. coli and $Y$. enterocolitica present in samples from tonsil surface and deep tonsil tissue and for the comparison of recoveries from the growth media CIN and YeCM. A single factor analysis of variance (ANOVA) was conducted for comparison of $E$. coli numbers detected in tonsils, faeces and on the carcass of ten pigs. A significance level threshold of $p<0.05$ was applied in general.

Most statistical tests related to the sequencing data were conducted in GraphPad Prism (version 7 , GraphPad Software Inc., La Jolla, CA, US). P-values calculated for multiple comparisons were adjusted by the False Discovery Rate (FDR) (Benjamini and Hochberg, 1995). A paired t-test was used for the overall comparison of the bacterial community composition on phylum level of samples from tonsil surface and deep tonsil tissue, while an unpaired t-test was used to compare the alpha diversity measures (Shannon index and species diversity) calculated for the two sample types. One-way ANOVA with multiple comparisons (Tukey's multiple comparisons test) was used to compare the alpha diversity measures (Shannon index and species diversity) calculated for the paired samples from tonsils, faeces and carcass swabs. The test was also used to assess the variation within and between different samples types and for the comparison of the mean relative abundance of specific genera in the paired samples from tonsils, faeces and carcass swabs. A oneway ANOVA of repeated measures and with multiple comparisons was used for comparison of the shared OTUs found in tonsils, faeces and on the three carcass swab areas. 
For the heatmap comparing the community on tonsil surface to that of tonsil tissue (Figure 5), pairwise comparisons of the $\log _{10}$ transformed relative abundances were conducted by use of permutation tests with 10.000 iterations and calculation of the FDR-adjusted $p$-values. These calculations were conducted in the software R (ver. 2.15.1, R Core Team 2014, Vienna, Austria) and RStudio (ver. 0.96.331) using in-house 242 scripts.

\section{Results}

\subsection{Culture analyses}

The bacterial community structure of tonsil surface and deep tonsil tissue was investigated in tonsils from 35 pig carcasses by both culture- and 16S rRNA gene sequencing-based analysis. The occurrence of $E$. coli and $Y$. enterocolitica on surface and in deep tissue of the tonsils is listed in Table 1. The number of $Y$. enterocolitica was found to be significantly higher in the deep tonsil tissue $(p<0.05)$ compared to surface. All Y. enterocolitica suspected colonies were identified as biotype 4, serotype 0:3. As no significant difference $(p=0.67$, paired t-test) was seen between the number of $Y$. enterocolitica found on $\mathrm{CIN}$ and $\mathrm{YeCM}$, only results from CIN are presented.

From the three different areas on the carcass, mean levels of $E$. coli and $Y$. enterocolitica were between 0.1 and $4.0 \mathrm{CFU} / \mathrm{cm}^{2}$, while the mean numbers in tonsils and faecal samples were 4.9 and $5.4 \log _{10} \mathrm{CFU} / \mathrm{g}$, respectively (Table 2). Significantly lower levels of $E$. coli were found on area B than on both area A and C. Y. enterocolitica was detected only in one faecal sample whereas seven tonsil samples had levels above the detection level (Table 2). Y. enterocolitica was not detected in any swab samples from area A, B and C by direct plating or following selective enrichment.

\subsection{Comparison of bacterial communities on tonsil surface and in deep tonsil tissue}

Analyses of the bacterial community structure by $16 \mathrm{~S}$ rRNA gene sequencing were performed with a mean sequencing depth of $27,671 \pm 12,234$ reads per sample. Samples were subsampled to 10,000 reads for further analysis. None of the samples from tonsil surface and deep tonsil tissue were, thereby, discarded 262 from the analysis.

263 At the phylum level, no significant differences in relative abundance between the six phyla found on tonsil 
consist of approximately $30-40 \%$ Proteobacteria, $30 \%$ Firmicutes, $20 \%$ Fusobacteria and $10-20 \%$ Bacteroidetes. The phyla Spirochaetes and Actinobacteria had mean relative abundances below $3 \%$. No significant difference in alpha diversity was seen between the surface of tonsils and the deep tonsil tissue (Shannon index; $p=0.59$ and observed species; $p=0.56$ ) (Figure 3a-b). Analysis of differences in bacterial community between sample types (beta diversity) by principal coordinate analysis (PCoA) based on unweighted UniFrac distances, indicated some separation between the two sample types (Figure 4a), which was supported by statistical analysis of the differences in UniFrac distances within and between sample types $(p<0.05)$ (Figure $4 b)$. The distribution of relative abundance of 63 different genera in tonsil surface samples and samples from deep tonsil tissue were visualised in a heatmap (Figure 5). Subsequent statistical analysis revealed 18 genera to be significantly different between the two sample types, including a significant higher relative abundance of Yersinia spp., and Pasteurella spp. in deep tonsil tissue compared to tonsil surfaces and significantly higher relative abundance of Actinobacillus spp. on tonsil surface. (FDR adjusted-p $<0.05)$.

\subsection{Comparison of bacterial communities in tonsils and faeces to communities in carcass swab} samples from 10 pigs

When using a cut-off value of 10,000 reads, two faecal samples and one carcass swab sample from area $A$ and B, respectively, were discarded from down-stream analysis. The remaining samples had an average sequencing depth of $28,440 \pm 11,710$ reads per sample.

The average distribution of phyla in the tonsil samples (Figure $2 \mathrm{~b}$ ) appeared similar to what was found in the 35 samples from tonsil surface and deep tonsil tissue (Figure 2a). The faecal samples showed a mean relative abundance of Firmicutes and Bacteroidetes of approximately $80 \%$ and $20 \%$, respectively, representing almost all sequences. The carcass swab samples showed increasing mean relative abundances of Proteobacteria from area C (50\%), to area B (71 \%) to area A (86 \%) while the relative abundance of both Firmicutes and Bacteroidetes were lowest in samples from area A (10\% and $2 \%$, respectively) and higher in samples from area B (15\% and $11 \%$, respectively) and highest in area C (27\% and $13 \%$, respectively). The mean relative abundance of Fusobacteria was also highest in samples from area C (6 \%). 
The alpha diversity (Shannon-index) showed an overall significant difference $(p<0.05)$ between the sample types with the diversity in sample area A found to be significantly lower than areas B and C (Figure 3c). No significant difference $(p=0.08)$ was seen between the richness of observed species in the different sample types (Figure 3d).

Principal coordinates analysis (PCoA) of the bacterial communities at the OTU level in tonsils, faeces and in the three different carcass swab samples showed clustering of the different communities (Figure 4c). In the PCoA plot, especially, the faecal and tonsil samples clustered separately while the carcass swabs clustered together. Visually, samples from area C clustered slightly closer to the tonsil samples compared to samples from the two other sample areas of the carcass. The single sample from the saw was included in the PCoA. Faecal samples clustered more closely together than other sample types, which is supported by low intrasample average distance (Figure 4d). No significant differences were found between mean distances within the tonsil samples and within samples from area A and B, respectively, while a significant difference was found between the tonsil samples and the samples from area $\mathrm{C}(p<0.05)$. A significant difference was also found between the mean distances within samples from area B and area $C(p=0.02)$. Although, the overall mean distances varied within the samples, average distances within all sample types were found to be significantly lower than the overall mean distances between all sample types $(p<0.05)$.

Looking at the distribution of OTUs shared between sample sites, we found that on average $43 \%$ of the OTUs present in tonsils were also present in samples from area C around the head (Figure $6 a$ ), $45 \%$ were found in samples from area A around rectum and around $30 \%$ were found in samples from area B along the backbone on the cut surface. Significantly less tonsil OTUs were shared with the OTU's from faecal samples compared to the sharing with the carcass swab samples $(p<0.05)$. From the faecal samples on average 48 $\%$ of the OTUs were found in samples from area A around the rectum, around $40 \%$ in samples from area B and $36 \%$ in samples from area C around the head (Figure 6b).

The relative distribution of the bacterial communities in the different sample types were visualised at the genus level in a heatmap (Figure 7). When compared to the other sample types, the tonsil samples showed higher relative abundance of genera such as Fusobacterium, Pasteurella, Moraxella and Actinobacillus. In the faecal samples we noted higher relative abundances for several of the genera belonging to the class Clostridia (Firmicutes) including Blautia and Roseburia. Among the three carcass areas, area C showed the 
highest relative abundance of Yersinia, Fusobacterium, and Actinobacillus but also most genera within the class Clostridia. Area A showed higher relative abundance of for Escherichia/Shigella compared to the two other areas. Overall, the sample from the saw showed low relative abundance of the phyla Actinobacteria, Bacteroidetes and Firmicutes and high relative abundances of genera within Proteobacteria. The sample from the saw e.g. showed the highest relative abundance of Salmonella and Escherichia/Shigella among all sample types. Significantly higher mean relative abundance of Yersinia was found in samples from area C compared to the other sample types $(p<0.05)$ (Figure 8a), while the mean relative abundance of Escherichia/Shigella in samples from area A was significantly higher than what was found in samples from tonsils, faeces and area B $(p<0.023)$ (Figure $8 b)$. The tonsils were observed to have a significantly higher mean relative abundance of Fusobacterium $(p \leq 0.05)$ compared to all the other sample types (Figure 8c).

\section{Discussion}

Tonsil surface and deep tonsil tissue showed overall comparable communities at the phylum level (Figure 2a). In contrast, when analysing at the more detailed genus level, 18 of the 63 compared genera were found to be significantly different in relative abundance, between the two sample types (Figure 5), which was supported by analysis of unweighted UniFrac distances (Figure 4a). The significant difference in relative abundance of Yersinia was consistent with the significantly higher number of $Y$. enterocolitica cultured in the deep tonsil tissue samples compared to tonsil surface. This is coherent with the ability of $Y$. enterocolitica to colonise the tonsils (Thibodeau et al., 1999). Lowe et al. (2012) also compared the bacterial communities from pig tonsil tissue and brush samples from the surface of tonsils. They primarily found differences between the communities in the species within the Enterobacteriaceae family and the obligate anaerobes, which they found more abundant in tissue samples compared to surface brush samples. This is essentially in accordance with our findings. When comparing surface associated bacteria with the deep tissue associated bacteria we did not apply surface disinfection of the tonsil surface before the analysis of the deep tissue community. A disinfectant applied on the surface may possibly have gained access to the bacterial community in the deeper tonsil tissue, thus biasing the community analysis. Also by our procedure, we cannot exclude that some bacteria, firmly attached to the surface, has been released during the second stomaching of the tonsil tissue, thus contributing to bias in the community analysis. Van Damme et al. 2018 compared the number of $E$. coli in tonsil surface to that of tonsil tissue and found similar levels of $E$. coli in tonsil tissue as in this study. In contrast to the present study, however, they recovered considerable lover 
numbers of $E$. coli by swabbing the surface than we did by stomaching. This could imply that we released more tissue associated E. coli into the surface homogenate in our samples. Despite this uncertainty, the microbiota analysis was able to disclose significant differences between tonsil surface and deep tissue associated bacteria at genus level. Collectively, we consider the employed procedure acceptable in relation to the conclusions of the study.

Faeces may also be a source of $Y$. enterocolitica contamination, but with much lower prevalence than seen for tonsils (Thibodeau et al., 1999). By culturing, we found $Y$. enterocolitica from only $10 \%$ of the faecal samples while it was found in $60-70 \%$ of the tonsil samples. This is in accordance with findings of Van Damme et al. (2013). A difference in carriage in faeces and in tonsils may be driven by a decrease in the proportion of pigs carrying human pathogenic $Y$. enterocolitica in faeces from about 135 days of age, while many of their tonsils do remain positive for Y. enterocolitica at the time of slaughter (Nesbakken et al., 2006). Accordingly, the tonsils may constitute a more important source of carcass contamination with human pathogenic $Y$. enterocolitica compared to faeces.

Escherichia coli can be present in large numbers both in tonsils (Fredriksson-Ahomaa et al., 2009) and in faeces (Nauta et al., 2013) and culturing of these organisms was included in the study in order to be able to compare the bacterial load of $E$. coli in the two sources (Table 2). However, both culturing and sequencebased analysis revealed no difference in load of Escherichia per gram between the two sources. The very high level of $E$. coli in tonsils may be due to ingestion of faeces.

If bacterial species are distributed differently in tonsils and faeces, their relative abundances may be used as an indicative marker for the source of carcass contamination. Even though $Y$. enterocolitica meet this criteria by being more abundant in tonsil, this study does not support it as a culturable marker for tonsil origin of carcass contamination as it was not detected on any of the swabbing areas by culturing.

In order to ease interpretation of results, the swabbing areas A, B and C were located on the inside of the carcass, as the inside is expected to be sterile until the carcass is cut open, and thus all bacteria present on raw muscle tissue must have originated from cross-contamination.

Very low levels of $E$. coli were recovered by culturing from the swabbing areas with significantly higher numbers found on area $A$ and $C$ around the rectum and the head, respectively compared to area $B$ on the 
mid part of the carcass. Thus, E. coli does not candidate as culturable marker for defining the relative contribution of faeces and tonsils as source of contamination.

By use of 16S rRNA gene sequencing it was possible to analyse the source of contamination in more depth. At phylum level we found the bacterial community of pig tonsils primarily to be dominated by Proteobacteria (30 - $50 \%$ ) in line with the "core microbiome" previously described by Lowe et al. (2012) although they found Fusobacteria less abundant. By culture-based analysis, Van Damme et al, (2018) supported this observation indirectly by finding Enterobacteriaceae, being part of Proteobacteria, more dominating in tonsils than in faeces. Faecal samples were vastly dominated by Firmicutes and Bacteroidetes (Figure 2b), which is consistent with previous studies by Kim et al. (2015) and Pajarillo et al. (2014). At phylum level the bacterial community of carcass area C mimicked the community of tonsil quite closely (Figure $2 \mathrm{~b}$ ). Like area $\mathrm{C}$, the areas A and B also contained a large proportion of Proteobacteria, but they did only contain a very small population of Fusobacteria. Even though the relative abundances of Bacteriodetes and Firmicutes in area $\mathrm{A}$ and $B$ are low, the almost absence of Fusobacteria indicate faeces to be the dominating source of area $A$ rather than the tonsils. At the same time, the high abundance of Proteobacteria indicate that a third source, not covered by this study, contribute to the carcarss contamination. This could be the process environment.

As carcasses are hanged with heads down, the community at lower parts could potentially be more diverse because the faecal community may blend in with the tonsil community at the forepart. A significant lower sequence diversity (lower alpha diversity (Shannon index)) in samples obtained from area A compared to those from area B and C (Figure 3c) supported this. Somewhat contradicting, however, no difference in the number of observed species between areas A, B and C were found (Figure 3d).

Investigating for systematic patterns in OTU sequence data by PCOA analysis, the carcass swab samples clustered together, with a tendency that samples from areas A and C clustered closer to the tonsil samples than area B (Figure 4c). This, together with the fact that around $45 \%$ of the OTUs present in the bacterial community of tonsils were also found in samples from area A and C (Figure 6a) could indicate that tonsils may play a role in contamination of both the forepart (area C) and of the ham area (area A). When comparing sequences from the ham area (area A) to sequences from faeces and tonsils almost $50 \%$ of the OTUs from area A were present in faecal samples while only approximately $25 \%$ were found in tonsil samples (Figure 6b). The latter indicate that area A mainly is contaminated with faeces but with evidence of 
a contribution from tonsils. The finding of $45 \%$ of tonsil OTU's in the ham region (area A) is likely a combination of contamination from tonsils and a high overlap between faecal and tonsil communities in general.

From the heatmap (Figure 7) it appears that tonsils compared to the other sample types, were dominated with genera within the family Pasteurellaceae (Actinobacillus, Haemophilus and Pasteurella) and the genera Moraxella, Alkanindiges as well as Fusobacterium. As these genera were part of the 'core microbiome' in porcine tonsils defined by Lowe et al. (2012) their presence could represent a general property of tonsils and potentially be indicative of contamination originating form tonsils. Contamination of the area $\mathrm{C}$ seems to be dominated by the flora from the tonsils, which is supported by a high relative abundance of Fusobacterium and Actinobacillus in tonsils and in area C, Actinobacillus is also detected from area A and B in low levels indicating tonsils as source of contamination (Figure 7).

The swabbing areas showed higher relative abundances of Enterobacteriaceae genera (other than E. coli) compared to samples from tonsils and faeces, which indicates a source of contamination not fully explained by faeces and tonsils as sources. If psychrotrophic Enterobacteriaceae establish and grow in the process environment, as may also be the case for the genus Aeromonas (Figure 7)., a subsequent transfer to the carcass, could potentially explain the increased occurrence of Enterobacteriaceae in swab samples The distribution of shared OTUs (Figure 6) also suggests a different source than faeces and tonsils to contribute to carcass contamination. The swab sample from the saw showed high relative abundances of several genera within the Enterobacteriaceae family and could be an example of process equipment where Enterobacteriacea dominates e.g due to growth.

The bacterial community composition of tonsils and faeces found in this study were similar to reports by Kim et al. (2015); Lowe et al. (2011, 2012), and Pajarillo et al. (2014). However, it is important to mention that differences in sample preparation, DNA extraction, and data handling can lead to differences in classification and may challenge the ability to compare sequencing results between studies. When specifically trying to compare culture-based cell counts for E. coli and Y. enterocolitica to the relative abundance of the corresponding genera obtained by sequencing, the detection limit for the sequencing method is also of great importance. Despite E. coli being detected in higher absolute numbers in faeces compared to carcass samples, the sequencing did not show a higher relative abundance of Escherichia/Shigella in faecal samples 
432 compared to the other samples (Figure 8b). This is most likely due to a higher total bacterial load in the 433 faecal samples and, thus, a higher detection limit for E. coli and lower deduced relative abundance when 434 sequencing.

435 The present study supports the hypothesis that it is possible to identify bacterial groups that are 436 characteristic for either tonsil or faecal carcass contamination. The level and composition of 437 Enterobacteriaceae on the carcasses indicates that other sources of contamination than tonsils and faeces 438 may be important. Future studies could include more samples from the working environment and process 439 equipment to provide a more in depth understanding of the specific sources. Our conclusions are based on 440 data from ten animals and future investigations will be needed to determine if the same systematic patterns 441 can be observed at population level. Inclusion of more slaughterhouses will also be relevant. If our findings 442 hold true on population level, it would enable a more general deduction of the sources behind bacteria found 443 on carcasses and may thus provide a more thorough basis for hygiene intervention at slaughter.

\section{Acknowledgements}

445 The present study was financed by the Danish Council for Strategic Research (DCSR, process 12-131417) 446 as a part of collaboration with the Strategic Research Collaboration in Food Science in the State of São 447 Paulo, Brazil and Denmark.

The Norwegian slaughterhouses are acknowledged for their kindness and help in relation to sampling. 
Table 1. Prevalence and mean number for Escherichia coli and Yersinia enterocolitica on the surface and in the deep tissue of tonsils ( $n=35)$ (Results for Yersinia only shown for CIN-agar).

\begin{tabular}{ccccccc}
\hline & \multicolumn{2}{c}{ Tonsil surface } & & \multicolumn{2}{c}{ Tonsil tissue } \\
\cline { 2 - 3 } \cline { 5 - 6 } & Prevalence & Mean concentration & & Prevalence & Mean concentration \\
& {$[\%]$} & {$[\log$ CFU/g] \pm sd } & & {$[\%]$} & {$[$ log CFU/g] \pm sd } \\
\hline E. coli & 100 & $5.5 \pm 0.6$ & & 100 & $5.6 \pm 0.4$ \\
Y. enterocolitica (CIN) & 70.7 & $3.5 \pm 1.2$ & & 68.3 & $4.2 \pm 0.9$ \\
\hline
\end{tabular}

449

450 
Table 2. Numbers of Escherichia coli (ECC) from faeces, tonsils and from swabbing areas A, B and C (see Figure 1), detected by use of the SimPlate method (AOAC International, 2005), and numbers of Yersinia enterocolitica (YeC) detected by culturing on CIN from the same sampling sites.

\begin{tabular}{|c|c|c|c|c|c|c|c|c|c|c|}
\hline \multirow{3}{*}{$\begin{array}{c}\text { Carcass } \\
\text { no. }\end{array}$} & \multirow{2}{*}{\multicolumn{2}{|c|}{$\frac{\text { Faeces }}{[\log \text { CFU/g] }}$}} & \multicolumn{2}{|c|}{ Area A } & \multicolumn{2}{|c|}{ Area B } & \multicolumn{2}{|c|}{ Area C } & \multicolumn{2}{|c|}{ Tonsils } \\
\hline & & & \multirow[b]{2}{*}{ ECC } & \multirow[b]{2}{*}{ YeC } & \multicolumn{2}{|c|}{$[\log$ CFU/cm²] } & \multirow[b]{2}{*}{ ECC } & \multirow[b]{2}{*}{ YeC } & \multicolumn{2}{|c|}{ [log CFU/g] } \\
\hline & ECC & YeC & & & ECC & YeC & & & ECC & YeC \\
\hline 1 & 4.8 & 4.91 & 0.27 & $<-0,2$ & -0.8 & $<-0,5$ & 1.7 & $<-0,2$ & 5.9 & 3.0 \\
\hline 2 & 4.8 & $<1,0$ & $<-1.2^{*}$ & $<-0,2$ & -1.5 & $<-0,5$ & 1.2 & $<-0,2$ & 4.5 & 3.0 \\
\hline 3 & 5.2 & $<1,0$ & -0.39 & $<-0,2$ & -1.3 & $<-0,5$ & 0.3 & $<-0,2$ & 4.9 & 1.8 \\
\hline 4 & 5.0 & $<1,0$ & -0.87 & $<-0,2$ & -1.5 & $<-0,5$ & -0.1 & $<-0,2$ & 5.1 & $<1.3$ \\
\hline 5 & 6.7 & $<1,0$ & $<-1.2$ & $<-0,2$ & -1.3 & $<-0,5$ & $<-1.2$ & $<-0,2$ & 4.2 & 1.6 \\
\hline 6 & $<4.0^{\star *}$ & $<1,0$ & $<-1.2$ & $<-0,2$ & $<-1.5$ & $<-0,5$ & $<-1.2$ & $<-0,2$ & 5.1 & $<1.3$ \\
\hline 7 & 5.7 & $<1,0$ & -0.57 & $<-0,2$ & -0.6 & $<-0,5$ & 0.8 & $<-0,2$ & 4.9 & $<1.3$ \\
\hline 8 & 6.9 & $<1,0$ & -0.03 & $<-0,2$ & -1.3 & $<-0,5$ & -0.4 & $<-0,2$ & 4.7 & $<1.3$ \\
\hline 9 & 5.9 & $<1,0$ & -0.27 & $<-0,2$ & $<-1.5$ & $<-0,5$ & -0.4 & $<-0,2$ & 4.1 & 3.6 \\
\hline 10 & 5.2 & $<1,0$ & $<-1.2$ & $<-0,2$ & $<-1.5$ & $<-0,5$ & -0.6 & $<-0,2$ & 5.9 & 4.7 \\
\hline Mean & $5.4^{\mathrm{a}}$ & & $-0.8^{b}$ & & $-1.4^{c}$ & & $-0.1^{b}$ & & $4.9^{a}$ & 2.2 \\
\hline $\mathrm{sd}^{\star \star \star}$ & 0.9 & & 0.6 & & 0.4 & & 1.0 & & 0.6 & 1.3 \\
\hline
\end{tabular}




\section{References}

Aliyu, S.H., Marriott, R.K., Curran, M.D., Parmar, S., Bentley, N., Brown, N.M., Brazier, J.S., Ludlam, H., 2004. Real-time PCR investigation into the importance of Fusobacterium necrophorum as a cause of acute pharyngitis in general practice. J. Med. Microbiol. 53, 1029-1035. doi:10.1099/jmm.0.45648-0

Anonymous, 2014. Commission Regulation (EU) No 218/2014 of 7 March 2014, amending Annexes to Regulations (EC) No 853/2004 and (EC) No 854/2004 of the European Parliament and of the Council and Commission Regulation (EC) No 2074/2005. Off. J. Eur. Communities.

AOAC International, 2005. Official Method 2005.03 Detection and Confirmed Quantitation of Coliforms and E. coli in Foods SimPlate Coliform and E. coli Color Indicator. Available at: http://www.aoac.org/omarev1/2005_03.pdf.

Benjamini, Y., Hochberg, Y., 1995. Controlling the False Discovery Rate: A Practical and Powerful Approach to Multiple Testing. J. R. Stat. Soc. Ser. B 57, 289-300.

Bokulich, N.A., Subramanian, S., Faith, J.J., Gevers, D., Gordon, J.I., Knight, R., Mills, D.A., Caporaso, J.G., 2012. Quality-filtering vastly improves diversity estimates from Illumina amplicon sequencing. Nat. Methods 10, 57-59. doi:10.1038/nmeth.2276

Borch, E., Nesbakken, T., Christensen, H., 1996. Hazard identification in swine slaughter with respect to foodborne bacteria. Int. J. Food Microbiol. 30, 9-25. doi:10.1016/0168-1605(96)00988-9

Caporaso, J.G., Kuczynski, J., Stombaugh, J., Bittinger, K., Bushman, F.D., Costello, E.K., Fierer, N., Peña, A.G., Goodrich, J.K., Gordon, J.I., Huttley, G. a, Kelley, S.T., Knights, D., Koenig, J.E., Ley, R.E., Lozupone, C. a, Mcdonald, D., Muegge, B.D., Pirrung, M., Reeder, J., Sevinsky, J.R., Turnbaugh, P.J., Walters, W. a, Widmann, J., Yatsunenko, T., Zaneveld, J., Knight, R., 2010. QIIME allows analysis of high- throughput community sequencing data. Nat. Methods 7, 335-336. doi:10.1038/nmeth0510-335

Edgar, R.C., 2010. Search and clustering orders of magnitude faster than BLAST. Bioinformatics 26, 24602461. doi:10.1093/bioinformatics/btq461 
Fredriksson-Ahomaa, M., Gerhardt, M., Stolle, A., 2009. High bacterial contamination of pig tonsils at slaughter. Meat Sci. 83, 334-6. doi:10.1016/j.meatsci.2009.06.004

Horter, D.C., Yoon, K.-J., Zimmerman, J.J., 2003. A review of porcine tonsils in immunity and disease. Anim. Health Res. Rev. 4, 143-155. doi:10.1079/AHR200358

Haas, B.J., Gevers, D., Earl, A.M., Feldgarden, M., Ward, D. V, Giannoukos, G., Ciulla, D., Tabbaa, D., Highlander, S.K., Sodergren, E., Methé, B., DeSantis, T.Z., Petrosino, J.F., Knight, R., Birren, B.W., 2011. Chimeric 16S rRNA sequence formation and detection in Sanger and 454-pyrosequenced PCR amplicons. Genome Res. 21, 494-504. doi:10.1101/gr.112730.110

Kim, J., Nguyen, S.G., Guevarra, R.B., Lee, I., Unno, T., 2015. Analysis of swine fecal microbiota at various growth stages. Arch. Microbiol. 197, 753-9. doi:10.1007/s00203-015-1108-1

Langworth, B.F., 1977. Fusobacterium necrophorum: its characteristics and role as an animal pathogen. Bacteriol. Rev. 41, 373-390.

Lowe, B.A., Marsh, T.L., Isaacs-Cosgrove, N., Kirkwood, R.N., Kiupel, M., Mulks, M.H., 2011. Microbial communities in the tonsils of healthy pigs. Vet. Microbiol. 147, 346-57. doi:10.1016/j.vetmic.2010.06.025

Lowe, B.A., Marsh, T.L., Isaacs-Cosgrove, N., Kirkwood, R.N., Kiupel, M., Mulks, M.H., 2012. Defining the "core microbiome" of the microbial communities in the tonsils of healthy pigs. BMC Microbiol. 12, 20. doi:10.1186/1471-2180-12-20

Mortensen, M. S., Brejnrod, A. D., Roggenbuck, M., Abu Al-Soud, W., Balle, C., Krogfelt, K. A., Stokholm, J., Thorsen, J., Waage, J., Rasmussen, M. A., Bisgaard, H., Sørensen, S. J. (2016). The developing hypopharyngeal microbiota in early life. Microbiome, 4(1), 70. doi:10.1186/s40168-016-0215-9

Nauta, M., Barfod, K., Hald, T., Sørensen, A.H., Emborg, H.-D., Aabo, S., 2013. Prediction of Salmonella carcass contamination by a comparative quantitative analysis of E. coli and Salmonella during pig slaughter. Int. J. Food Microbiol. 166, 231-7. doi:10.1016/j.ijfoodmicro.2013.07.014 
Nesbakken, T., Eckner, K., Høidal, H.K., Røtterud, O.J., 2003. Occurrence of Yersinia enterocolitica and Campylobacter spp. in slaughter pigs and consequences for meat inspection, slaughtering, and dressing procedures. Int. J. Food Microbiol. 80, 231-240.

Nesbakken, T., Iversen, T., Eckner, K., Lium, B., 2006. Testing of pathogenic Yersinia enterocolitica in pig herds based on the natural dynamic of infection. Int. J. Food Microbiol. 111, 99-104. doi:10.1016/j.ijfoodmicro.2006.04.019

Pajarillo, E.A.B., Chae, J.P., Balolong, M.P., Kim, H.B., Seo, K.-S., Kang, D.-K., 2014. Pyrosequencingbased analysis of fecal microbial communities in three purebred pig lines. J. Microbiol. 52, 646-51. doi:10.1007/s12275-014-4270-2

Schloss, P.D., Westcott, S.L., Ryabin, T., Hall, J.R., Hartmann, M., Hollister, E.B., Lesniewski, R.A., Oakley, B.B., Parks, D.H., Robinson, C.J., Sahl, J.W., Stres, B., Thallinger, G.G., Van Horn, D.J., Weber, C.F., 2009. Introducing mothur: open-source, platform-independent, community-supported software for describing and comparing microbial communities. Appl. Environ. Microbiol. 75, 7537-41. doi:10.1128/AEM.01541-09

Sundberg, C., Al-Soud, W.A., Larsson, M., Alm, E., Yekta, S.S., Svensson, B.H., Sørensen, S.J., Karlsson, A., 2013. 454 pyrosequencing analyses of bacterial and archaeal richness in 21 full-scale biogas digesters. FEMS Microbiol. Ecol. 85, 612-626. doi:10.1111/1574-6941.12148

Thibodeau, V., Frost, E.H., Chenier, S., Quessy, S., 1999. Presence of Yersinia enterocolitica in tissues of orally-inoculated pigs and the tonsils and feces of pigs at slaughter. Can. J. Vet. Res. 63.

Thorsen, J., Brejnrod, A., Mortensen, M., Rasmussen, M. A., Stokholm, J., Al-Soud, W. A., Sørensen, S., Bisgaard, H., Waage, J. (2016). Large-scale benchmarking reveals false discoveries and count transformation sensitivity in 16S rRNA gene amplicon data analysis methods used in microbiome studies. Microbiome, 4(1), 62. doi:10.1186/s40168-016-0208-8

Tulstrup, M.V.-L., Christensen, E.G., Carvalho, V., Linninge, C., Ahrné, S., Højberg, O., Licht, T.R., Bahl, M.I., 2015. Antibiotic Treatment Affects Intestinal Permeability and Gut Microbial Composition in 
Wistar Rats Dependent on Antibiotic Class. PLoS One 10, e0144854. doi:10.1371/journal.pone.0144854

528 529 530 531 532 533 534 535 536 537 538 539

Van Damme, I., Berkvens, D., Baré, J., De Zutter, L., 2013. Influence of isolation methods on the occurrence of plasmid-carrying Yersinia enterocolitica serotype 0:3 in slaughter pig tonsils, faeces and carcass surface swabs. Int. J. Food Microbiol. 164, 32-35. doi:10.1016/j.ijfoodmicro.2013.03.019

Van Damme, I., mattheus, W., Bertrand, S., Zutter, L.D. (2018), Quantification of hygiene indicators and Salmonella in the tonsils, oral cavity and rectal content samples of pigs during slaughter. Food Microbiol. 71, 120-128

Wauters, G., Kandolo, K., Janssens, M., 1987. Revised biogrouping scheme of Yersinia enterocolitica. Contrib. Microbiol. Immunol. 9, 14-21.

Yu, Y., Lee, C., Kim, J., Hwang, S., 2005. Group-specific primer and probe sets to detect methanogenic communities using quantitative real-time polymerase chain reaction. Biotechnol. Bioeng. 89, 670-679. doi:10.1002/bit.2034 
Figure 1. Illustration of the three swabbing areas A, B and C. Source: www.21food.com.

Figure 2. Bacterial community composition in samples at phylum level. Mean relative abundance of the phyla; a) found in samples from tonsil surface and tonsil tissue, b) in samples from faeces, area A, area B, area C (see location in Figure 1) and tonsil surface.

Figure 3. Alpha diversity of bacterial communities calculated as Shannon index $(a, c)$ and number of observed OTUs (b,d) in samples of tonsil surface and deep tonsil tissue (a-b) and in samples from tonsils, faeces, and carcass areas A, B and C (c-d). Columns show means with error bars indicating standard error of means (SEM). Asterisks indicate significant differences, ${ }^{\star \star \star \star}, p<0.0001 ; \mathrm{ns}$, not significant.

Figure 4. Principal Coordinates Analysis (PCoA) plots (unweighted UniFrac distances) $(a, c)$ and mean

554 555 556

Figure 5. Heatmap of relative abundance of bacterial genera for individual samples obtained from tonsil surface $(n=35)$ and tonsil tissue $(n=32)$. Colours indicate $\log _{10}$ relative abundance. A colour indication of e.g. -2 means an abundance of $1 \%$ of the total community. Only bacterial genera with $35 \%$ prevalence across all samples are included. Asterisks indicate significant differences, with red indicating higher relative abundance in tonsil surface and green indicating higher abundance in tonsil tissue (FDR corrected permutation based t-test); ${ }^{*}, p<0.05 ;{ }^{*}, p<0.01$. The left-hand colour bar shows the classification of bacterial genera (yellow: Actinobacteria, red: Bacteroidetes, cyan: Bacilli (Firmicutes), dark blue: Clostridia (Firmicutes), light blue: Erysipelotrichia (Firmicutes), purple: Fusobacteria, salmon: $\beta$-Proteobacteria, lightgreen: $\varepsilon$-Proteobacteria, dark-green: $ү$-Proteobacteria, orange: SR1, Spirochaetes and Tenericutes).

\section{Figure 6. Mean fraction of those OTUs identified in tonsils (a) and faeces (b) also found in other} compartments including area A (around the head), area B (along the backbone on the cut surface) and area $C$ (around the head). Columns show means with error bars indicating standard error of means (SEM). Asterisks indicate significant differences (FDR-adjusted $p$-values); $n s$, not significant; ${ }^{*}, p<0.05 ;{ }^{* *}, p<0.01$. 
574 Figure 7. Heatmap of relative abundance of bacterial genera in individual from tonsils $(n=10)$, faeces $(n=8)$ 575 and carcass swabs from area $A(n=9), B(n=9)$ and $C(n=10)$. Colours indicate $\log _{10}$ relative abundance.. 576 Only bacterial genera with $35 \%$ prevalence across all samples are included. The left-hand colour bar shows 577 the classification of bacterial genera (yellow: Actinobacteria, red: Bacteroidetes, cyan: Bacilli (Firmicutes), 578 dark blue: Clostridia (Firmicutes), light blue: Erysipelotrichia (Firmicutes), purple: Fusobacteria, salmon: $\beta$ 579 Proteobacteria, light-green: $\varepsilon$-Proteobacteria, dark-green: $\gamma$-Proteobacteria, orange: SR1, Spirochaetes and 580 Tenericutes).

582 Figure 8. Relative abundance of Yersinia (a), Escherichia/Shigella (b) and Fusobacterium (c) in samples 583 from tonsils, faeces and area A, B and C. Columns show mean values with error bars indicating standard 584 error of means.

585 
587 Figure 1

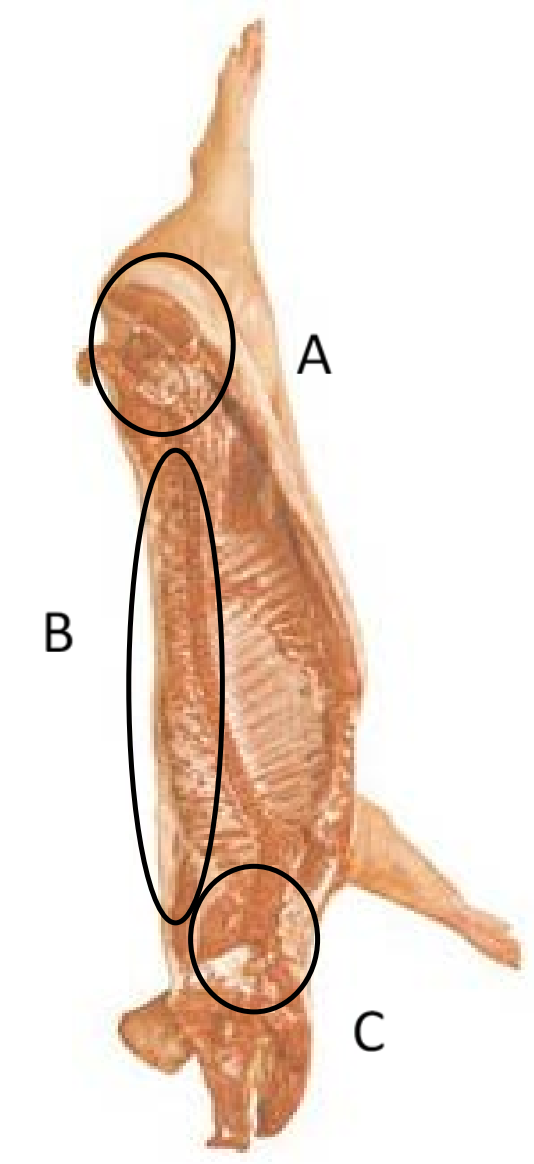

588

589 
Fig. 2

a

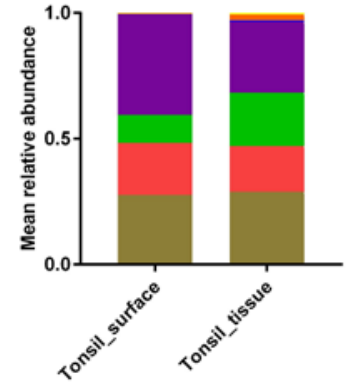

b

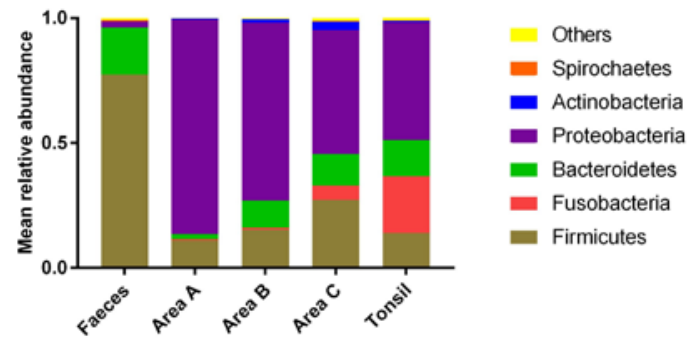

Fig. 2 


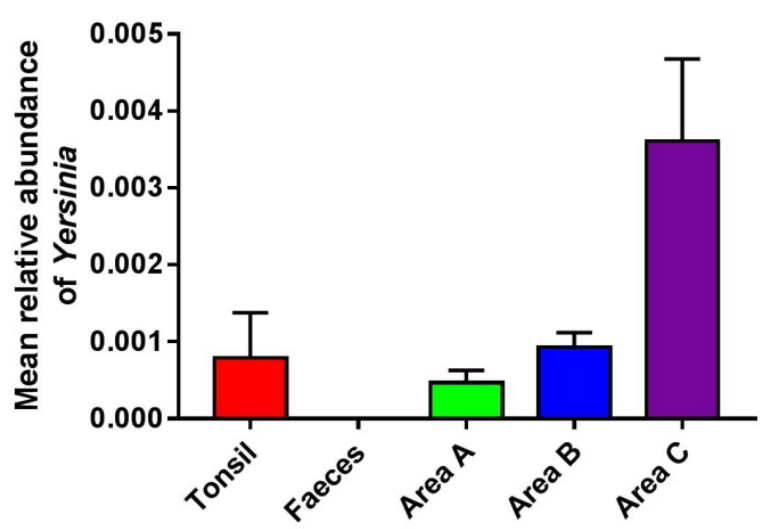

C

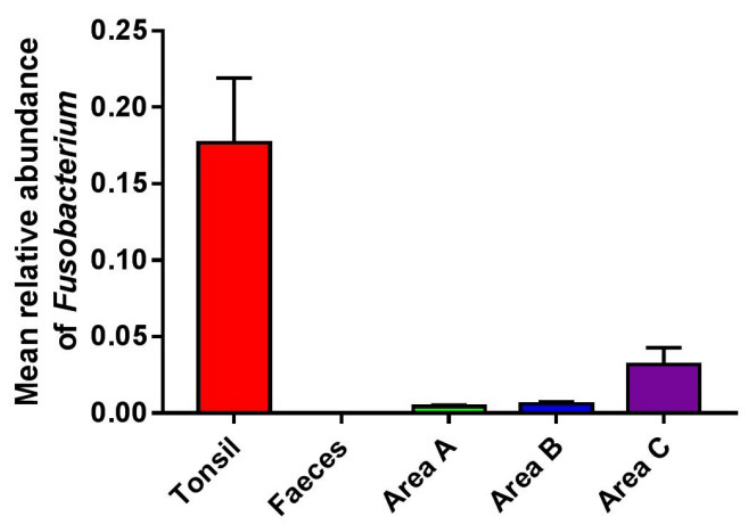

b

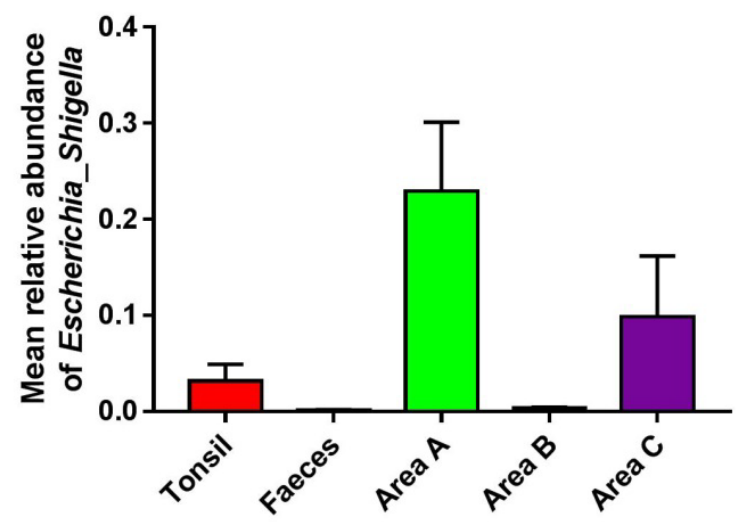

601

602

603 
a

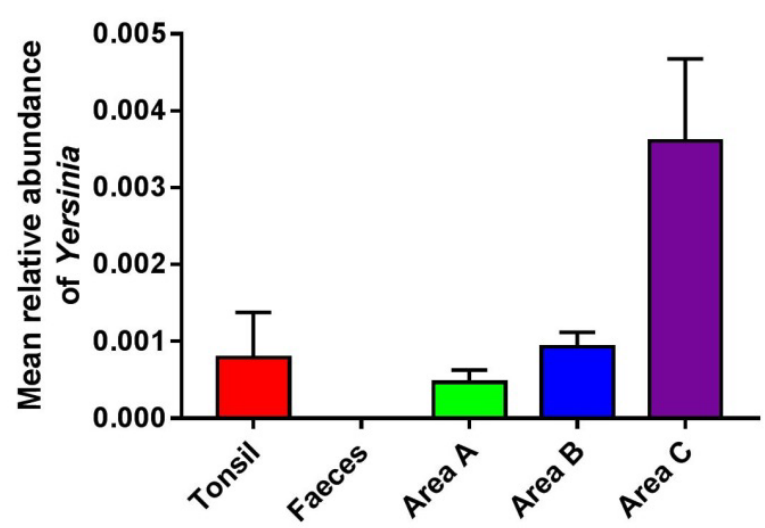

C

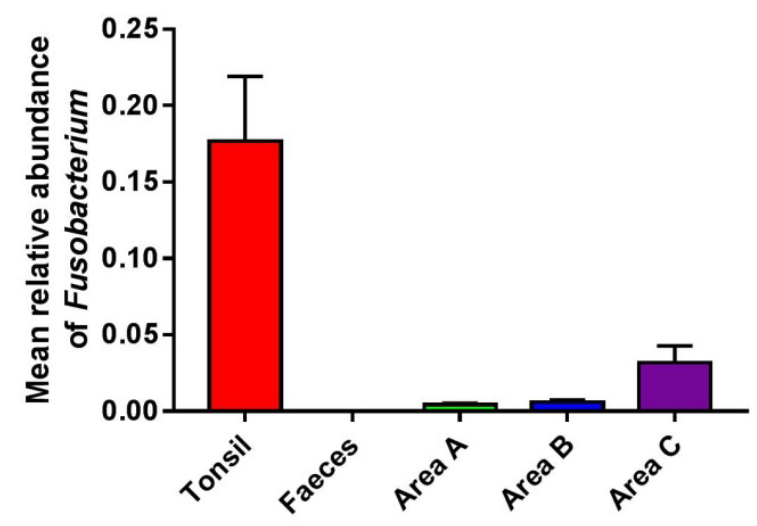

b

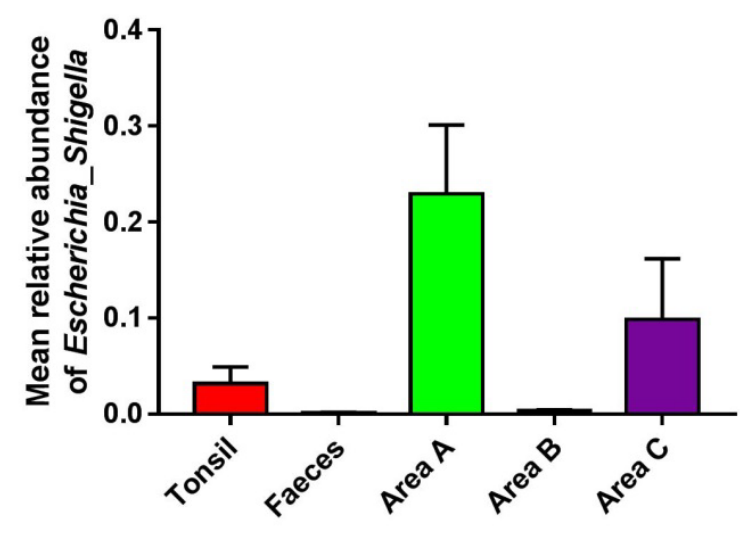

605

606 


\section{Figure 5}

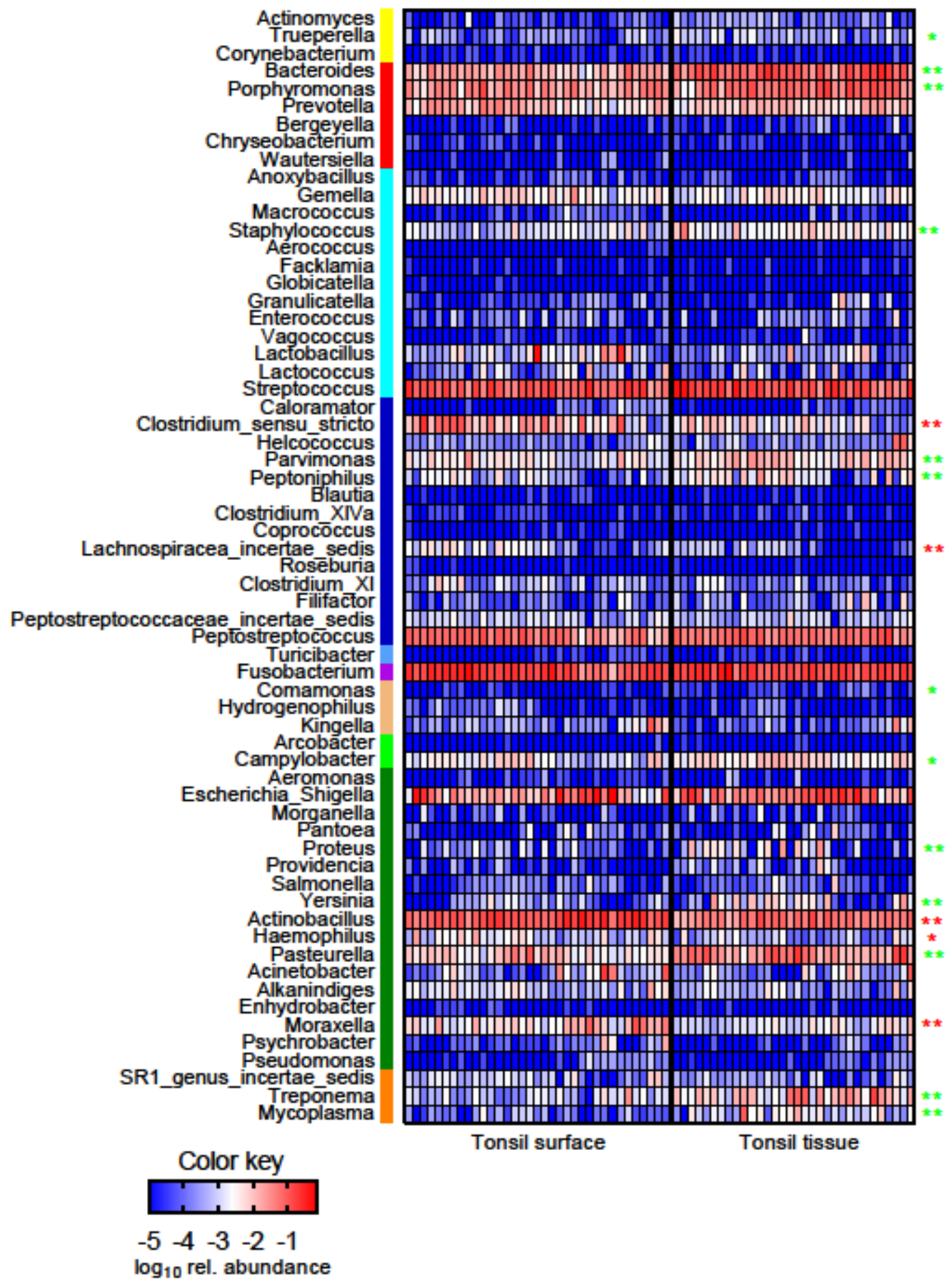

Figure 5 
611 Figure 6

612
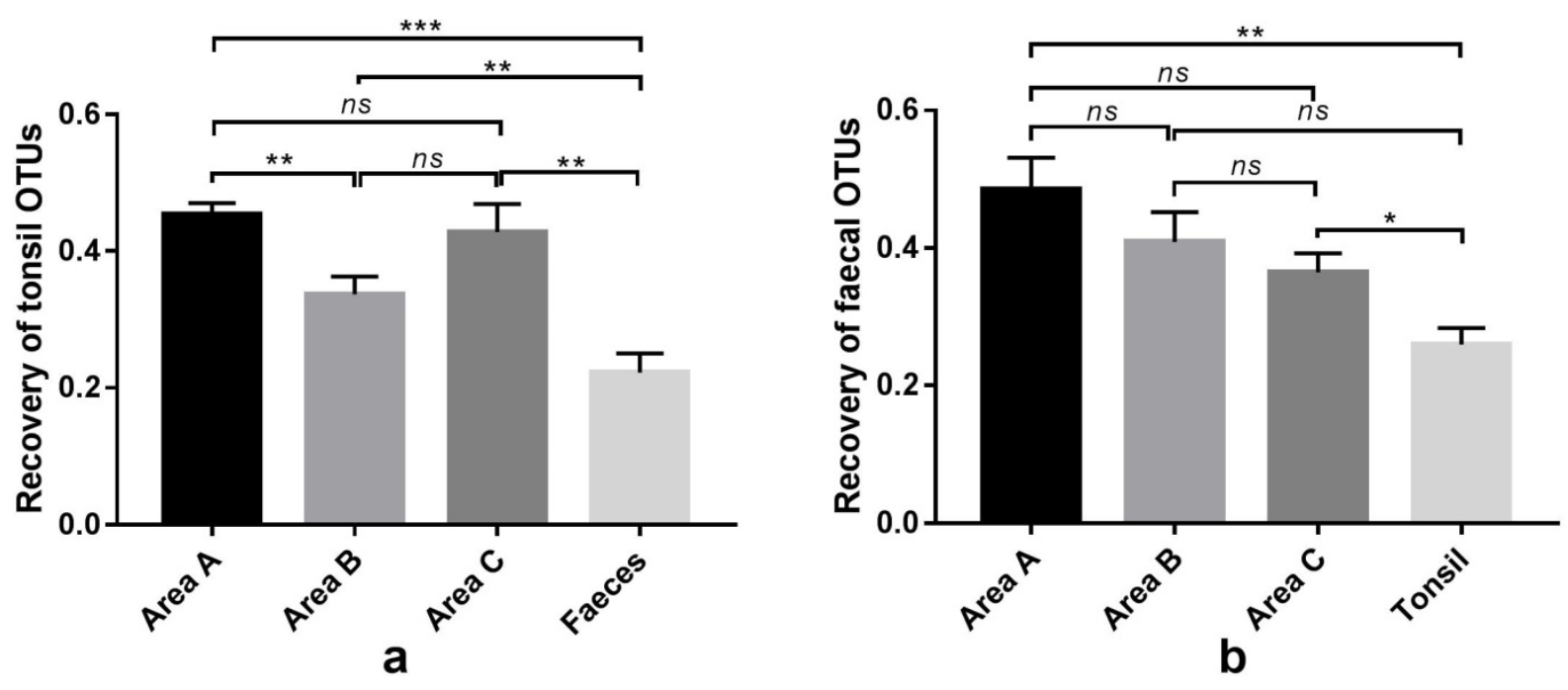


\section{Figure 7}

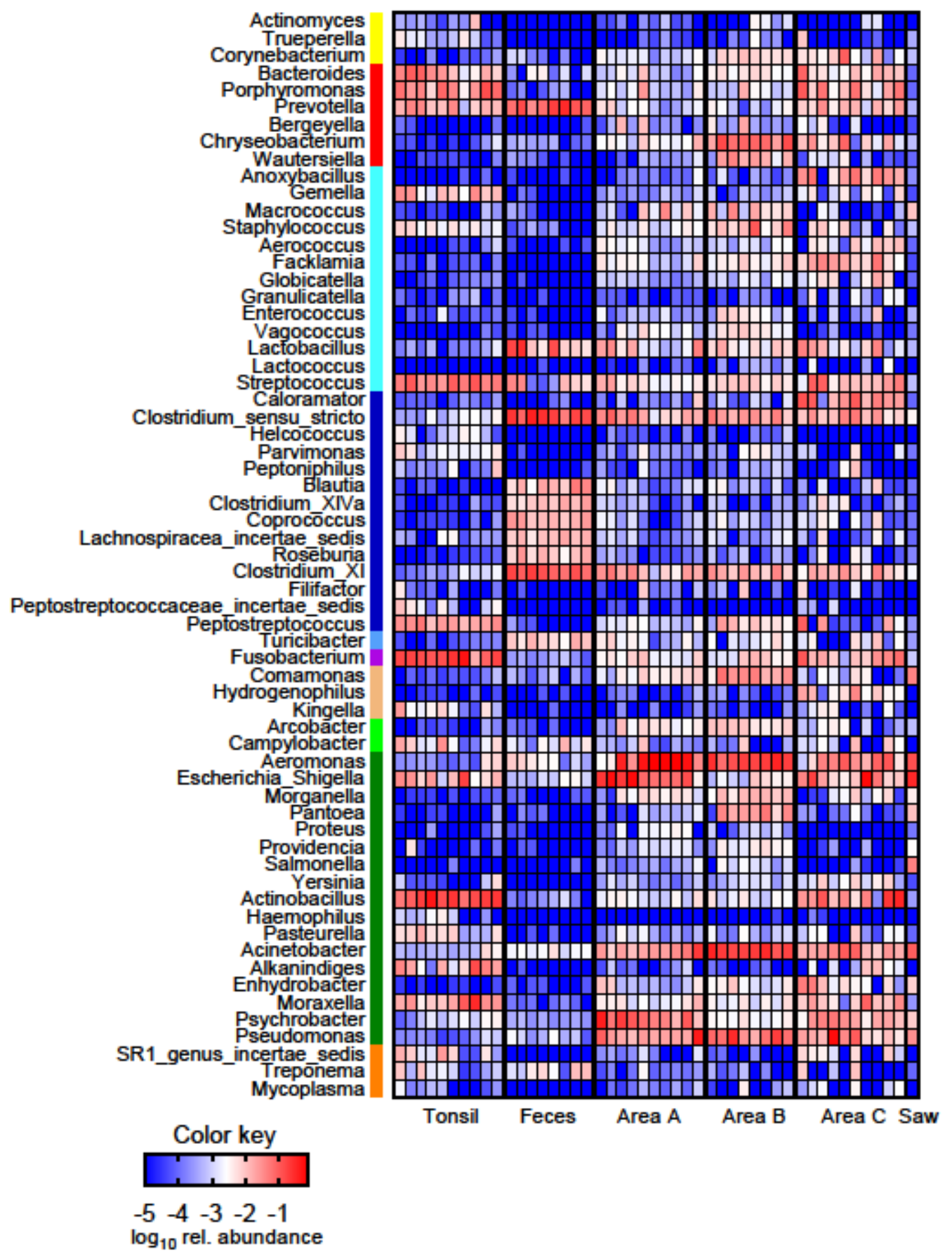

Figure 7 
Figure 8

a

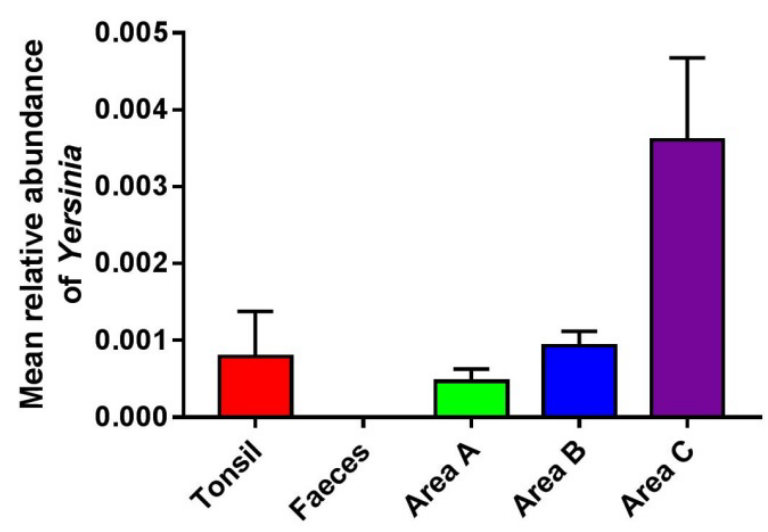

c

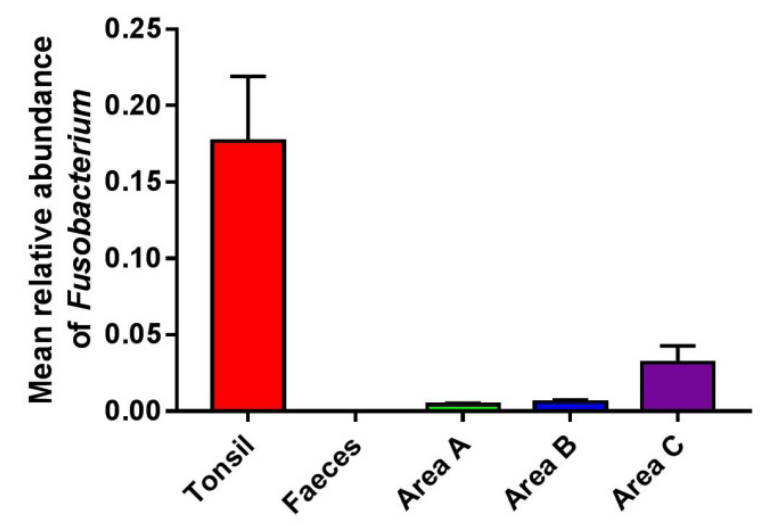

b

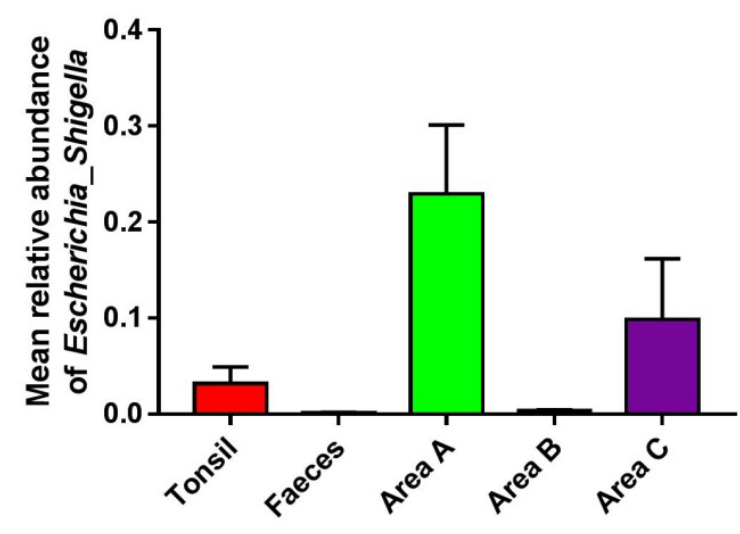

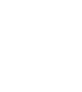


Highlights

- Major phyla in pig tonsils were Proteobacteria and Fusobacteria

- Clear differences in microbiota at genus level between tonsil surface and deep tonsil tissue

- The genera Moraxella, Fusobacterium, and Actinobacillus are potential markers of carcass contamination from tonsils 